Revue musicale OICRM

\title{
Isabelle Ronzier « à la croisée des chemins »
}

\section{Sophie Renaudin}

Volume 7, numéro 2, 2020

URI : https://id.erudit.org/iderudit/1072419ar

DOI : https://doi.org/10.7202/1072419ar

Aller au sommaire du numéro

\section{Éditeur(s)}

Observatoire interdisciplinaire de création et recherche en musique (OICRM)

\section{ISSN}

2368-7061 (numérique)

Découvrir la revue

\section{Citer ce document}

Renaudin, S. (2020). Isabelle Ronzier « à la croisée des chemins ». Revue musicale OICRM, 7(2), 111-124. https://doi.org/10.7202/1072419ar

\section{Résumé de l'article}

Médiatrice culturelle indépendante, Isabelle Ronzier retrace dans cet entretien son parcours et évoque les différents milieux artistiques dans lesquels elle a évolué - la musique, la danse, le théâtre, mais aussi l'écriture et la marionnette. Son expérience et ses réflexions nous amènent à concevoir la médiation comme une forme créatrice, tant pour celle ou celui qui la met en oeuvre que pour ceux qui la partagent. Cet article est ainsi l'occasion de découvrir les différents formats de médiation mis en place par Isabelle Ronzier et les multiples projets qu'elle a réalisés. Pour compléter ce portrait, l'annexe propose trois documents qui présentent, sous différents formats (notes et photographies), plusieurs étapes du projet de création Le pont sur le monde (2017-2019). 


\title{
Isabelle Ronzier «à la croisée des chemins »
}

\author{
Sophie Renaudin
}

\begin{abstract}
Résumé
Médiatrice culturelle indépendante, Isabelle Ronzier retrace dans cet entretien son parcours et évoque les différents milieux artistiques dans lesquels elle a évolué - la musique, la danse, le théâtre, mais aussi l'écriture et la marionnette. Son expérience et ses réflexions nous amènent à concevoir la médiation comme une forme créatrice, tant pour celle ou celui qui la met en œuvre que pour ceux qui la partagent. Cet article est ainsi l'occasion de découvrir les différents formats de médiation mis en place par Isabelle Ronzier et les multiples projets qu'elle a réalisés. Pour compléter ce portrait, l'annexe propose trois documents qui présentent, sous différents formats (notes et photographies), plusieurs étapes du projet de création Le pont sur le monde (2017-2019).
\end{abstract}

Mots clés : création ; écriture ; médiation culturelle ; pédagogie ; Isabelle Ronzier.

\begin{abstract}
In this interview, Isabelle Ronzier, an independent cultural mediator, recounts her career path and talks about the different artistic backgrounds in which she has evolved-music, dance, theatre but also writing and puppetry. Her experience and her thoughts lead us to apprehend mediation as a creative form, both for the person who implements it and for those who share it. This article is thus an opportunity to discover the different forms of mediation set up by Isabelle Ronzier and the many projects she has carried out. To complete this portrait, the appendix offers three documents that present several stages of the creative project Le pont sur le monde (2017-2019).

Keywords: creation; cultural mediation; pedagogy; Isabelle Ronzier; writing.
\end{abstract}


Médiatrice culturelle indépendante et musicienne intervenante, Isabelle Ronzier travaille en tant que chargée de mission pour les Jeunesses musicales de France et pour 1'Opéra Grand Avignon en collaboration avec le Conservatoire à Rayonnement Régional (CRR) du Grand Avignon et l'association culturelle pour le jeune public Le тотем. Depuis 2009, elle est également chargée de cours sur les questions liées à la médiation culturelle auprès des étudiants du CFMI (Centre de formation pour les musiciens intervenants) d'Aix-Marseille Université. En plus de ces activités, Isabelle Ronzier anime des ateliers d'écriture créative dans les musées d'Avignon. Ses compétences ne s'arrêtent pas là, puisqu'elle est également biographe d'artistes ${ }^{1}$. Pour elle, ces activités multiples se regroupent en une seule et même posture : celle d'être à l'écoute des personnes qui l'entourent et d'être en capacité de capter leur parole.

Je l'ai rencontrée le temps d'un après-midi, à Avignon, et nous avons pu évoquer ensemble son parcours et son expérience comme "médiatrice culturelle». Les premiers moments de notre rencontre ont d'ailleurs permis d'expliciter les enjeux derrière le choix de ces mots qu'elle emploie couramment pour qualifier une activité professionnelle qu'elle a initialement principalement exercée dans le domaine musical.

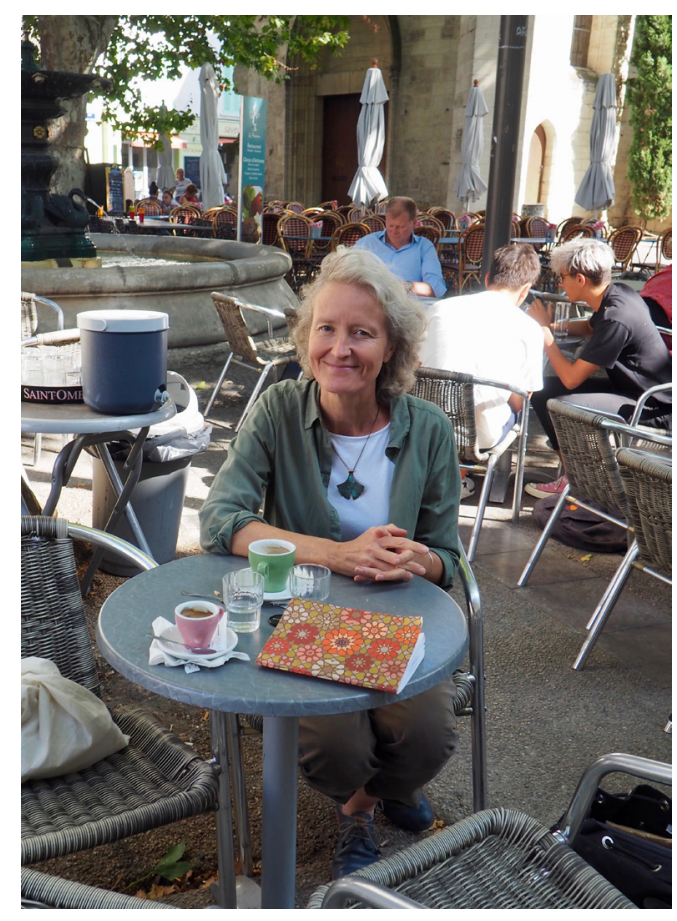

Figure 1 : Rencontre avec Isabelle Ronzier autour d'un café sur la place des Corps Saints à Avignon.

Photo : Sophie Renaudin, septembre 2019.

$1 \quad$ En 2016, Isabelle Ronzier se forme au métier de biographe d'artiste pour s'y consacrer dans les années à venir. Dans cette perspective, elle retrace le parcours d'une femme partie en Sibérie dans les années 1980 à la rencontre des communautés koriaques dans une première biographie qui s'intitule Le chant de Grand Corbeau. Parcours d'une missionnaire en Sibérie à la fin du régime soviétique. En plus de rendre compte du parcours de vie des artistes, Isabelle Ronzier s'intéresse à la vie des lieux habités et transformés par les artistes qui s'y installent. C'est vers ce concept de "biographie de lieux de vie culturels » que s'oriente désormais Isabelle Ronzier. Son deuxième ouvrage, intitulé Les mémoires de Berdine, est en cours de rédaction et porte sur l'histoire de la Bergerie de Berdine, lieu de vie culturel et artistique situé en France dans le Lubéron. 
Isabelle Ronzier : Je me présente professionnellement comme médiatrice culturelle. [...] Souvent, on me demande d'expliquer ce que cet intitulé signifie. On a davantage en tête l'image du médiateur dans le cadre juridique, comme quelqu'un qui règle les conflits, plutôt que la conception du médiateur comme interface entre un certain nombre de personnes et d'évènements ou d'objets. [...] Pour l'identifier, le terme à accoler au mot " médiation " est le terme "culturel ", parce que la médiation s'adresse à des objets de culture au sens large. La médiation peut s'apposer à tous les contextes, à toutes les esthétiques de la culture, de quelque nature qu'elles soient. Pour moi, les processus de la médiation culturelle en général sont les mêmes que ceux de la médiation musicale en particulier. La médiation de la musique demande des compétences précises dans le domaine musical, avec tant d'esthétiques et d'approches différentes qu'on se retrouve face à une démultiplication des pratiques, comme dans toute pratique culturelle.

Je dirais que la médiation est une posture, un désir de créer du lien et de mettre en lien des personnes, des pratiques artistiques - ou culturelles - et des objets, par le biais de différents moyens qui mettent ces personnes en situation de création. La médiation est un acte de création, au sens très large du terme : c'est une posture de création, aussi bien pour la personne qui mène la médiation que pour la personne qui la vit et qui l'expérimente. Cette notion de mise en relation et d'interface peut être résumée par une expression qui était l'objet de mon mémoire de musicienne-intervenante et pour laquelle j'ai toujours eu une certaine tendresse : être « à la croisée des chemins ${ }^{2} » \ldots$

Sophie Renaudin : Alors on fait appel à toi pour la médiation d'objets musicaux et pour des objets culturels au sens large...

I. R. : Jusqu'à présent, on faisait essentiellement appel à moi pour de la musique. C'est lié à mon parcours personnel : j'ai toujours évolué dans ce milieu-là, tout en évoluant dans d'autres domaines, comme la danse, la marionnette et l'écriture. Je me suis aussi ouverte à la pratique de la médiation par l'écriture créative ; depuis quelques années, je pratique la médiation dans les musées autour des œuvres d'art. [...] La médiation dans les musées consiste à mettre en place des dispositifs qui permettent de tisser un lien entre le public et le lieu, avec l'histoire du lieu, avec le bâtiment, et, au-delà, avec la ville. Dans un musée, tout peut faire objet de médiation - aussi bien les personnes qui ont fondé le musée que celles qui l'ont traversé : des auteurs, des photographes, mais aussi des objets, des œuvres, des documents d'archive... Quand la médiation s'occupe des œuvres elles-mêmes, ce peut être autant l'œuvre que le cartel ou un détail de l'œuvre. Il y a une infinité d'approches possibles. Dans la médiation de la musique, j'ai souvent été amenée à utiliser autre chose que la musique elle-même : des tableaux de musiciens, leur écriture, leur graphie, leur signature, leur témoignage ou bien leur parole. Et je pense que cela s'applique à tous les domaines... du moins, tous les domaines artistiques. Je n'ai pas encore fait de médiation autour de la cuisine... (Rires.)

2 Isabelle Ronzier (2003), Le musicien intervenant, un artiste pédagogue à la croisée des chemins, mémoire du diplôme de musicien-intervenant en milieu scolaire, Université de Provence Aix-Marseille. 
$\mathrm{Au}$ fil de la discussion, Isabelle Ronzier me raconte sa formation, au cours des années 1980. Il n'existe alors pas de formation de médiateur, même si le contexte politique, notamment avec l'arrivée de François Mitterrand et de Jack Lang au pouvoir, favorise une certaine impulsion dans le domaine de l'éducation artistique et culturelle ${ }^{3}$. Sa formation principale est musicale : après plusieurs années de cours privés de flûte baroque et de stages de musique ancienne, Isabelle Ronzier entre en octobre 1982 dans la classe de musique ancienne au Conservatoire de Strasbourg. Au cours de ses années de formation, elle fait la rencontre d'une troupe de danse baroque et décide de suivre cette compagnie et de travailler pour elle à Paris. Elle évolue ainsi dans une nouvelle sphère artistique, qui mêle danse et musique. Parallèlement, elle entre à l'École nationale supérieure des arts et techniques du théâtre (ENSATT) en régie, son et lumière, afin de se former de manière professionnelle à la mise en scène. En plus de la danse et de la musique, c'est désormais le cinéma qui la passionne : elle décide de commencer une licence d'études cinématographiques et audiovisuelles à Jussieu. Elle alterne alors entre un travail à l'Opéra en régie et un poste d'assistante à la mise en scène dans la compagnie de danse baroque. Les postes qu'elle occupe appartiennent principalement au domaine de l'assistanat (assistante au metteur en scène, à la chorégraphe...), posture qui, pour elle, est équivalente à celle du médiateur. Comprendre la question de l'organisation du plateau et de la salle, saisir les attentes du metteur en scène, celles des artistes, les entendre et y répondre : autant de compétences mobilisées qui définissent la posture choisie par Isabelle Ronzier et qui témoignent d'un véritable souci de "l'autre ». Cette posture correspond également à un modèle démocratique $d u$ " faire ensemble », elle témoigne d'une volonté de reconnaitre une capacité d'agir aux participants et traduit une volonté de mettre en place un processus de création collective d'un objet musical - culturel - ou d'un projet.

À partir de ma rencontre avec Isabelle Ronzier, j'ai souhaité dresser son portrait de " médiatrice culturelle » en le composant en trois parties. D'abord, il s'agit de s'intéresser aux deux éléments qui fondent la personnalité d'Isabelle Ronzier, et qui l'ont guidée vers le domaine de la médiation : l'écoute et l'amour du son. Puis, nous aborderons les liens entre médiation et pédagogie qui transparaissent dans ses multiples activités. Enfin, il s'agira de saisir avec précision, à travers des exemples de réalisation d'activité de médiation, la dimension créative indissociable du métier de médiatrice tel qu'il est pensé par Isabelle Ronzier.

\section{L'ÉCOUTE ET LE SON}

Les diverses expériences vécues par Isabelle Ronzier ont été déterminées par le lien intime qu'elle entretient avec la musique, et plus particulièrement avec le son. Auprès de la compagnie de danse, elle collabore avec des musiciens baroques ; dans son

3 Sur ce sujet, voir les travaux de Philippe Poirrier (pour une synthèse de l'histoire des politiques culturelles en France voir notamment Histoire des politiques culturelles de la France contemporaine, Dijon, Bibliest, Université de Bourgogne, 1998) et ceux de Jean Caune (La culture en action, De Vilar à Lang. Le sens perdu, Grenoble, Presses Universitaires de Grenoble, 1992). 
master à Jussieu, elle travaille sur les bandes-son et différentes illustrations sonores au cinéma. Pour le cinéma, elle écrit également pendant un temps les doublages français de films anglophones, poste rémunéré qui mêle écriture et travail sur le son et qui lui permet de mener plusieurs activités artistiques en parallèle. Plus tard, c'est à la radio qu'elle s'intéresse : entre 1986 et 1990, elle a l'opportunité de réaliser des reportages et des créations radiophoniques qui sont diffusés sur les grandes radios publiques françaises. Parmi ces créations, trois sont primées dans la catégorie documentaire au concours de création radiophonique Phonurgia Nova ${ }^{4}$.

I. R. : En filigrane, j'ai toujours aimé ce qui était lié au son, à la radio [...] j'ai toujours adoré enregistrer les gens et capter leurs histoires pour la restituer. Comprendre ce qu'ils sont, mettre en valeur ce qu'ils font. Pour moi, c'est aussi une posture de médiateur [...] J'ai toujours considéré le reportage ou le documentaire comme une action de médiation, avant d'être un travail de reportage. Et à l'inverse, je retrouve cette dimension créative, celle des reportages ou des documentaires, dans mon métier de médiateur.

La musique, le son et la création sonore sont les trois éléments moteurs du parcours d'Isabelle Ronzier. L'attention à la voix, qu'elle soit parlée, chantée, prononcée à la radio, ou recueillie dans un contexte singulier, et l'attachement au son, sont deux aspects de sa personnalité qui fondent sa posture de médiatrice. L'attention à la voix pour et en elle-même d'une part, et l'attention constante à la voix des autres, d'autre part. Être à l'écoute : voilà l'attitude qui définirait l'éthique de la médiatrice qu'est Isabelle Ronzier.

I. R. : Plusieurs fois, on m'a demandé : mais pourquoi ne diriges-tu pas une structure? Non, ça ne m'intéresse pas... Je pense que c'est quelque chose que 1'on partage en tant que médiateurs. On est attaché à l'attention à l'autre et à la mise en valeur de l'autre. Si l'on est amené à avoir des postures de direction, c'est toujours dans une forme de coordination collective ou collaborative.

Cette attitude d'écoute, indispensable dans le métier de médiateur pour établir un dialogue avec les personnes auxquelles on s'adresse, est également une compétence recherchée lors de la conception de l'activité de médiation, au moment de la discussion avec les partenaires qui sont impliquées dans l'activité. Isabelle Ronzier décrit cette dimension politique du métier de médiateur telle qu'elle l'a vécue en tant que chargée de mission pour les orchestres de région de Montpellier puis d'Avignon.

4 Par exemple : «Des cerfs-volants dans le ciel » et « À la rencontre des marionnettes » sont deux créations primées qui portent respectivement sur le festival du cerf-volant de Dieppe et sur le festival du théâtre d'objets de Fontblanche à Vitrolles. Les deux documentaires de création ont été diffusés sur France Culture dans l'émission Clair de nuit. Isabelle Ronzier est également l'auteure de pièces radiophoniques comme « Le fauteuil 27 », une adaptation du roman d'Harry Dickson diffusée en 1986 dans l'émission Tentative première sur France Inter. 
I. R. : La médiation est très liée au développement territorial, ainsi qu'à la recherche de financements et à la politique. Un orchestre de région est financé par un certain nombre de collectivités, et chaque collectivité est en demande d'actions qui relèvent de sa compétence. Il y a donc un cahier des charges. Il faut comprendre ce qu'une collectivité demande, et ce dont elle a besoin pour pouvoir y répondre... Il faut alors saisir les attendus de chacun (de la ville, du département, de la région, de l'État) et se positionner par rapport à eux, mettre en place les dispositifs de création et comprendre ce que sont les attendus des musiciens d'orchestre et des artistes. Tout est très lié : le politique, le développement territorial, la médiation et l'artistique.

\section{PÉDAGOGIE ET MÉDIATION}

Isabelle Ronzier entremêle pédagogie et médiation au sein de ses différentes activités. Elle a été professeure de musique dans plusieurs collèges ${ }^{5}$, et au lycée autogéré de Paris. Dans ce cadre, elle a proposé aux lycéens dont elle avait la charge de participer au projet "Dix mois d'école et d'Opéra ", un des premiers dispositifs emblématiques de médiation à l'Opéra Bastille ${ }^{6}$. Il était question d'amener les enfants sur le plateau, de leur faire expérimenter les décors, les costumes... Et de raconter une histoire au public en créant la bande-son du spectacle à partir d'un montage d'extraits d'opéras.

Son engagement vis-à-vis du milieu scolaire ne s'arrête pas là : en tant que formatrice auprès des étudiants du CFMI, Isabelle Ronzier est chargée de cours pour l'analyse du milieu des écoles dans lesquelles interviennent les étudiants, s'occupe de leur insertion professionnelle et les forme aux actions de médiation menées en partenariat avec une structure culturelle. Isabelle Ronzier est également référente pédagogique des Jeunesses musicales de France.

s. R. : Comment conçois-tu l'importance de la pédagogie dans la médiation? Qu'est-ce qui différencie les deux et qu'est-ce qui les rapproche?

I. R. : La pédagogie pose la question de savoir comment partager une expérience avec les publics. Par la connaissance, la pratique, la rencontre? En tant que pédagogues, nous sommes toujours amenés à nous poser ces questions : qu'est-ce qu'on transmet à nos élèves et comment? De quoi leur parle-t-on ? Par quels moyens et par quels outils ? La pédagogie est un rapport à la transmission. En tant que professeur, on est aussi médiateur : d'une part, entre le sujet que l'on traite et le groupe des élèves, d'autre part, entre le sujet et chaque élève du groupe.

5 Équivalent français de l'école secondaire québécoise.

6 Sur ce sujet, voir l'article de Suzana Kubik (2019), «Dix mois d'école et d'opéra. Un premier pas vers l'art lyrique ", 13 septembre, https://www.francemusique.fr/savoirs-pratiques/dix-mois-ecole-et-dopera-76269, consulté le 2 décembre 2019. La page du site de l'Opéra Bastille consacrée à ce projet offre également de nombreux renseignements ; il est notamment possible de consulter le rapport commandé par l'Académie de l'Opéra national de Paris sur l'impact de "Dix mois d'école et d'opéra » sur les élèves ayant bénéficié de ce dispositif entre 2003 et 2018 : https://www.operadeparis.fr/academie/education-artistique/dix-mois-decole-et-dopera, consulté le 2 décembre 2019. 
En tant que médiateur, il est important de former les responsables pédagogiques qui accompagnent les publics dans l'expérience de la médiation. Se pose alors la question des outils pédagogiques que l'on met à leur disposition, afin, qu'à leur tour, ils puissent préparer leur public. En tant que chargée de médiation pour les orchestres de région de Montpellier et d'Avignon, j'ai mené un important travail de réflexion sur les dossiers pédagogiques. Comment rédiger et mettre en forme un outil de médiation dont les publics puissent se saisir en autonomie, tout en donnant des pistes de pratique et d'appropriation des contenus au-delà de l'expérience du concert ou du spectacle ? C'est cette expérience rédactionnelle qui m'a amenée à devenir référente pédagogique des JM France et à coordonner un comité de rédaction d'une dizaine d'auteurs, musicologues, conseillers pédagogiques en musique et musiciens intervenants en milieu scolaire.

\section{CRÉER ET IMMERGER LES PUBLICS DANS LA CRÉATION}

Après avoir été chargée de mission en milieu scolaire à 1'Orchestre de Montpellier (2003-2009), Isabelle Ronzier est recrutée par 1'Orchestre régional Avignon-Provence pour créer le service des nouveaux publics et de médiation culturelle. Dans ce cadre, elle mène plusieurs actions de médiation en lien avec l'orchestre dans les écoles d'Avignon. En mobilisant et en explorant le répertoire de musiques contemporaines, ces actions engagent les enfants et les adolescents dans des expériences créatives dont ils sont pleinement acteurs.

Depuis 2015, elle travaille en indépendante, et a développé trois projets de création et de médiation en milieu scolaire pour 1'Opéra du Grand Avignon dans le cadre d'un dispositif d'éducation artistique et culturelle menée à l'échelle de la collectivité en partenariat avec l'association Le TOTEM/Éveil artistique et le CRR du Grand Avignon. Chaque projet, mené sur deux années scolaires, permet la rencontre et la collaboration entre élèves d'une part, et musiciens intervenants, musiciens interprètes, compositeurs et artistes de spectacle vivant d'autre part, pour la composition et la production d'une œuvre qui mêle musiques (jazz, musiques du monde ou hip-hop) et autres disciplines artistiques (théâtre, cirque, danse...). Cette création est ensuite représentée sur le plateau de l'Opéra Grand Avignon. Deux reportages sur les deux premières actions de création et de médiation avec 1'Opéra sont disponibles en ligne : Les p'tits loups du jazz font leur cirque à l'opéra et Le pont sur le monde. Pour le deuxième projet intitulé Le pont sur le monde, Isabelle Ronzier a reçu le Prix de l'enseignement musical 2019, remis par la Chambre syndicale des Éditeurs de Musique de France ${ }^{7}$. Une autre preuve de la proximité des liens entre médiation, enseignement et création.

Face à l'incroyable diversité des formats de médiation mis en place par Isabelle Ronzier, émerge la question des formes idéales de médiation.

7 Voir le palmarès des prix de l'année 2019, disponible à cette adresse : http://www.cemf.fr/lpem/ les-prix-de-lenseignement-musical-2019-le-palmares/, consultée le 2 décembre 2019. 
I. R. : C'est une question de parcours personnel. En tant que musicienne intervenante en milieu scolaire, je crois que la forme idéale de médiation est celle de la création. Quand le public est immergé dans la pratique, il expérimente lui-même la posture et la rencontre avec les artistes, en étant artiste lui-même. Je pratique cette forme de médiation depuis que j'ai commencé à travailler pour les orchestres symphoniques. Cela induit tout un parcours : depuis la formation des pédagogues qui encadrent les enfants et la formation des musiciens intervenants, jusqu'à 1'expérience du spectacle partagé avec les artistes professionnels sur scène, en passant par la rencontre entre les enfants et les artistes, entre les enfants et les différents corps de métier du spectacle, de la mise en scène à la régie...

Je pense également très mobilisateur de faire participer les publics à l'action de médiation : les solliciter pour organiser un évènement avec eux : c'est par exemple ce que j'ai fait avec des associations d'étudiants de l'Université d'Avignon et avec des lycéens. À Avignon, les étudiants ont pris en charge l'organisation de concerts de l'orchestre au sein de l'Université, depuis la gestion des répétitions et l'installation technique jusqu'à la communication et l'accueil des artistes et de la communauté universitaire. C'est une forme de médiation dans laquelle les publics sont leurs propres médiateurs.

D'autres actions de médiation plus classiques et plus simples à organiser consistent à faire rencontrer les publics avec les artistes : les interventions en milieu scolaire ou dans différents lieux, sous forme de concert-intervention, par exemple. C'est de la médiation ponctuelle, les artistes se déplacent auprès des publics ou bien les publics se déplacent auprès des artistes - dans le cas des répétitions générales par exemple. En amont, ces activités impliquent la formation des enseignants, la rédaction de dossiers et la mise à disposition d'outils pédagogiques.

Enfin, j'ai développé depuis quelques années la médiation par l'écriture créative. Je pense que l'atelier d'écriture est un outil de médiation extraordinaire pour mettre les personnes en situation de création, pour restituer leur ressenti en sollicitant à la fois la mémoire et les émotions, dans une pratique à la fois individuelle et collective. J'ai commencé cette pratique pour répondre à la demande de l'association Musique baroque en Avignon d'un cycle de médiations liées aux différents concerts donnés sur une saison. Je faisais écrire les personnes en amont autour des œuvres au programme, des répertoires, des lieux où se donnaient les concerts, des artistes qui les interprétaient. Puis, les publics étaient invités à venir écouter, parfois rencontrer les artistes. Dans ce type de médiation, les publics sont créateurs, dans une autre discipline que celle de l'objet de la médiation.

S. R. : Quelle est ton implication dans une activité de médiation en tant que médiatrice? Comment te définis-tu?

I. R. : Quand je travaille avec les étudiants du CFMI sur la posture de médiateur lors d'actions de sensibilisation autour d'un spectacle musical, je développe avec eux l'idée selon laquelle il existe trois types d'interactions avec les publics sans valeur hiérarchique entre eux. Le premier type est celui de la médiation didactique : on transmet une connaissance, on raconte quelque chose, les publics sont en posture d'écoute et de mémorisation. La forme caractéristique est celle de la conférence. 
Le deuxième type est celui de la médiation interactive : le public est invité à se positionner, à porter un regard, à s'exprimer ou encore à débattre sur ce qu'il ressent, sur ce qu'il a expérimenté. La forme caractéristique est celle de l'échange entre les artistes et les publics à l'issue du spectacle en bord de plateau. Le troisième type est celui de la médiation créative : le public expérimente les processus de composition des œuvres au programme, ou bien entre dans un dispositif d'improvisation et de création avec l'artiste ou le musicien intervenant [...].

Cela fait peu de temps que j'ai pris conscience que ma posture de médiatrice était artistique. La médiation est la forme créative par laquelle je m'exprime. Par exemple, dans le dispositif Les Arts sur le Pont créé pour l'Opéra d'Avignon, j'ai un statut de directrice artistique déléguée, indépendante des institutions qui m'ont missionnée. Aujourd'hui, je m'autorise à concevoir chaque projet comme une création, dont toutes les dimensions sont artistiques, depuis la recherche de subventions auprès des partenaires qui m'accompagnent depuis plusieurs années, jusqu'au choix des artistes et à l'écriture du spectacle, en passant par la rédaction des outils de médiation et de communication...

Pour les actions d'écriture créative, je me revendique comme créatrice de projets de médiation. Finalement, je réalise que le rêve de devenir metteuse en scène qui m'a fait choisir d'entrer dans une école de théâtre par la porte de la régie technique au début de ma carrière professionnelle a fini par se réaliser dans la médiation : mettre en scène différents éléments artistiques entre eux à l'intention d'un public.

ET POUR LA SUITE...

S. R. : Des projets, en cours ou à venir ?

I. R. : Pour le dispositif Les Arts sur le Pont, j'ai réussi à faire travailler plusieurs institutions entre elles, à faire collaborer une dizaine d'artistes, à mobiliser le réseau d'éducation nationale, avec le soutien des collectivités territoriales et des fondations. Je considère ce projet comme l'aboutissement de ma pratique de médiatrice institutionnelle. J'ai eu beaucoup de retours, de témoignages, de dessins ; le retour des enfants montre quelque chose d'assez magnifique ${ }^{8}$.

Je crois que s'ouvre une nouvelle période de médiation qui passera par l'écriture avec la pratique de biographe et l'accompagnement des artistes dans leurs récits de vie. Actuellement j'aide une personne dans la rédaction des mémoires d'un lieu de vie sociale dans le Lubéron, La Bergerie de Berdine. J'expérimente ce qu'est écrire l'histoire d'un projet. Dans le même esprit, j'aimerais beaucoup contribuer aux mémoires de lieux de création et de démarches artistiques : mettre en valeur les projets, les artistes et les publics par l'écriture. 
Ces documents sont extraits du dossier dans lequel Isabelle Ronzier a regroupé la présentation et le bilan du projet Le pont sur le monde. Ce projet fait partie du dispositif Les Arts sur le Pont, dispositif d'éducation artistique et culturelle mis en œuvre par l'Opéra Grand Avignon et porté par Le тотЕM/Éveil Artistique, scène conventionnée jeune public, et par le CRR du Grand Avignon. Le projet a été développé pendant deux années scolaires, en 2017-2018 et 2018-2019, auprès des élèves de huit écoles du Grand Avignon, autour de la musique du monde et des chants bulgares. La première année a été consacrée à la découverte et à la pratique du répertoire par les élèves avec les musiciens-intervenants en milieu scolaire ; la deuxième a été dédiée à la création et la production du spectacle. À partir de ces rencontres avec les enfants et à partir du répertoire choisi, le compositeur Sylvain Griotto a composé l'œuvre intitulée Le pont du monde pour chœur et marionnettes. L'œuvre a été créée sur la scène de l'Opéra Confluence en juin 2019.

\section{DOCUMENT 1}

Restitution de la deuxième rencontre entre les enfants de l'école Marie Curie (Le Pontet), le compositeur Sylvain Griotto, et les musiciens intervenants.

La rencontre a eu lieu le jeudi 27 septembre 2018. Notes prises par Isabelle Ronzier, productrice artistique déléguée des Arts sur le Pont.

\section{Le Pontet, Anne-Laure Ouhayoun}

École Marie Curie, $14 \mathrm{~h}$

$1^{\text {er }}$ groupe, deux classes

Nous avons partagé le repas avec Boris, Anne-Laure, Laure et Sylvain. Convivialité des échanges, l'équipe se construit autour de ces repas improvisés.

Laure et Sylvain font connaissance, croisent leurs expériences, leurs postures artistiques, leurs démarches de création.

Nous sommes entrés dans la salle polyvalente, deux classes, cela fait un grand groupe. La masse d'enfants est beaucoup plus importante, à la différence des classes de ce matin.

Sylvain : «On va inventer notre façon à nous de faire de la musique bulgare à partir des chants que vous avez appris. On va voir si on est prêts à faire de la musique ensemble. On oublie les temps de récré, de repas. On fait des sons ensemble. »

Sylvain invite les enfants à murmurer leur prénom en boucle. Par un geste de la main, il guide le jeu vocal collectif.

La masse vocale murmurée produit un ronronnement de machine.

" Ça va devenir de plus en plus fort ». Sylvain amplifie le geste. Les enfants automatiquement chantent plus fort. Le geste se réduit, le son redescend un niveau en dessous. Les enfants sont très réactifs et répondent vocalement au quart de tour aux gestes de Sylvain. 
Grand groupe d'enfants, grande densité de regard.

Laure se place derrière les enfants, elle sent qu'ils la regardent et qu'ils se demandent qui elle est.

On se présente avec Laure et Boris, les enfants peuvent se concentrer à nouveau sur Sylvain, «Tout ce qu'on va faire ensemble va prendre une forme. »

Les enfants écoutent Sylvain avec beaucoup d'attention.

Il suggère l'idée de vagues, de vagues fortes, de vagues douces. Pour faire des vagues, on a besoin de respirer de prendre de l'air.

À 50, ce son grave produit une texture magnifique, profonde, intense.

Encore moins fort, plus secret. Puis plus fort.

"Je peux vous faire faire tous les sons de la terre. Les sons ce sont comme des êtres humains. La concentration c'est apprendre à faire des sons sans rire, même si cela peut être drôle. » Il responsabilise les enfants sur l'attention portée au son qu'ils produisent.

Les enfants jouent le jeu, les yeux pétillants, un petit sourire au coin des lèvres, tout en restant concentrés. Ils sont heureux de répondre à la demande de Sylvain, ils ont confiance en lui.

Maintenant qu'il a acquis leur confiance, il peut jouer avec eux, les faire entrer dans sa musique.

Il garde lui-même un visage souriant et bienveillant. Les enfants entrent dans sa complicité.

La main de Sylvain devient une palette qui sculpte les sons et leur donne une forme rythmique, une forme sonore, une texture.

Petit à petit, Sylvain emmène les enfants vers la partition Hubava Rada.

Il demande à Anne-Laure de prendre son violon. Il intègre Anne-Laure dans l'intervention, il sait que c'est la première fois que les enfants la revoient depuis l'année dernière. Il joue avec la réalité temporelle des enfants et des intervenants.

Moment très émouvant, entre le chant des enfants et le chant du violon. Une belle façon pour les enfants de reprendre contact avec leur musicienne intervenante.

Sylvain traduit le chant en français. «Les paroles sont tristes ? Non. Alors pourquoi le chantez-vous de façon triste?"

Un enfant répond : "Elle est triste parce qu'elle est obligée de faire le ménage avant d'accueillir son amoureux ».

"Les Bulgares ont cette particularité de chanter avec une certaine nostalgie, ils peuvent aussi chanter des histoires joyeuses de façon triste et des histoires tristes de façon joyeuse. Finalement, on ne sait pas. On peut mélanger la joie et la tristesse. »

Sylvain lit les textes écrits par les mamans. Partage de souvenirs sur la joie et la tristesse mêlées. Choix de trois vers issus des textes :

- Aïda : J'ai de la peine, mais tout ira bien,

- Lindita : La vie est belle, mais aussi difficile,

- Marwoua : Ma force c'est de transformer mes larmes en eau qui s'éloigne. 
Proposition faite à la maîtresse : écrire d'autres phrases qui soient à la fois tristes et joyeuses.

Sylvain dit la phrase d'Aïda en russe et travaille la langue avec les enfants. Travail sur la prononciation, l'intonation, le rythme. Les enfants jouent sérieusement avec Sylvain et avec la phrase en russe. Sylvain libère le rire des enfants.

Puis il reprend l'apprentissage de la phrase, en jeux vocaux, par imitation.

Sylvain demande à Anne-Laure de jouer un ré corde à vide en continu. Ils choisissent de mettre la sourdine pour que le son ne couvre pas les voix des enfants.

Les enfants sont invités à dire le texte à voix basse en s'appuyant sur le continuo du ré, avec un changement d'archet à chaque note.

On rentre dans la fabrication de musique.

Sylvain entraîne les enfants dans l'invention d'une mélodie. Il donne des indications de jeu à Anne-Laure pour suggérer des climats harmoniques, avec les notes de la mélodie de Hubava rada. Cinq hauteurs de son : ré-mi-fa-sol-la.

Jouer avec les notes, les mettre dans l'ordre au choix.

Un enfant essaye, il reste sur la même note ré-ré-ré-ré-ré-ré-ré. Reprise par les enfants en grand groupe, en travaillant la couleur Un deuxième enfant : ré-mi-fa-mi-ré-do.

Un troisième : ré-mi-fa-mi-mi-ré. Question-réponse.

"On est les compositeurs? ", demande un enfant.

"Bien sûr", répond un autre enfant.

"L'idée d'Inès était une bonne idée, on presse cette idée comme un citron pour qu'elle nous en donne d'autres. »

Les enfants expérimentent différentes propositions, ils se rendent compte à quel point il est complexe de donner une idée, d'explorer de nouvelles pistes, de développer des idées, d'assumer son idée.

Un enfant fait une dernière proposition.

Sylvain : "c'est celle que j'attendais, avec une petite modulation. »

Les enfants s'approprient peu à peu le jeu. Ils se lèvent à la demande de Sylvain, ils vont chanter ce qu'ils viennent d'explorer.

Les enfants sont nommés par leur prénom, chacun ayant contribué à l'élaboration de la ligne mélodique. Travail de l'intonation pour faire surgir la surprise mélodique (la note «bleue »).

Réglage du bourdon et de la ligne mélodique à l'octave avec Anne-Laure Sylvain, qui choisit quatre propositions mélodiques.

La partition s'élabore en passant d'une classe à l'autre, chaque classe contribuant à une partie du puzzle, et transmettant au reste des chœurs d'enfants sa part de création. L'œuvre se construit à partir des différents éléments du puzzle d'écriture. 
"Vous savez ce qu'est un bourdon?»Oui, répond un enfant, c'est un son qui reste toujours le même. Sylvain : " un bourdon c'est comme un mat auquel tout le monde peut s'accrocher. »

"On a recueilli des idées musicales qui vont me permettre de composer. Je vais fabriquer de la musique à partir de la matière que vous m'avez donnée. On est dans la phase d'élaboration du matériau musical. »

\section{DOCUMENT 2}

Répétition des enfants, lumière sur le plateau de 1'Opéra
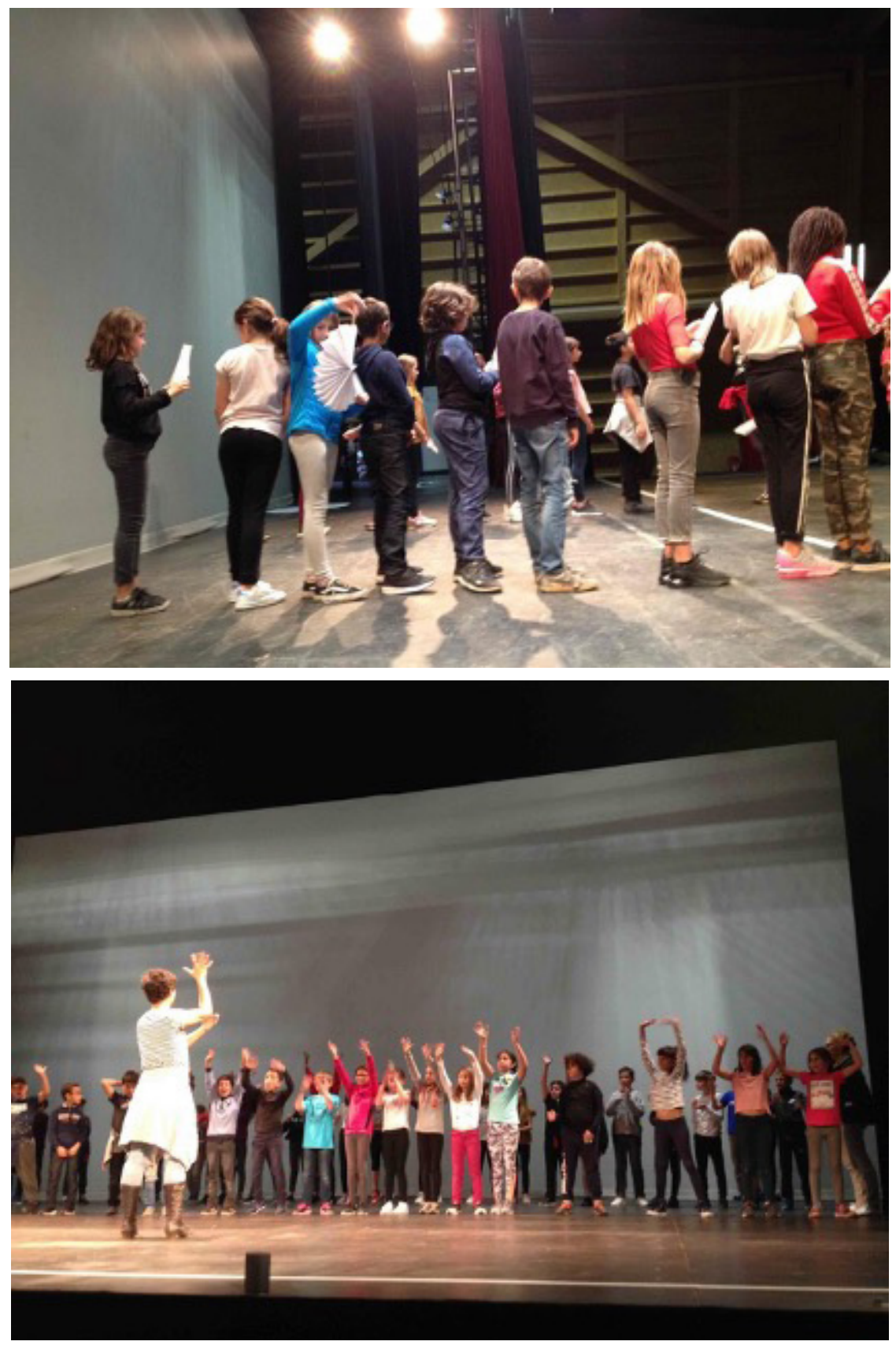


\section{DOCUMENT 3}

IMPRESSIONS des élèves de CM1 de la classe de Mme FAUX - École de SAZE Création du spectacle Le pont sur le monde 6, 7 et 8 juin 2019 Opéra Confluence

Ça fait deux ans que j'attends ce spectacle. J'ai ressenti de la joie et de la fierté d'être là. J'avais un petit peu le trac devant tout ce monde. (Léo)

Le spectacle, ça s'est passé plus vite que dans les répétitions. J'ai aussi ressenti qu'on était de vrais, mais de vrais choristes. Puis on chantait plus vite et il y avait plus d'ambiance que dans les répétitions. (Ethan)

J'ai adoré ce projet. J'ai bien aimé Cati, Sylvain, Zoé et le groupe AKSAK. Surtout que c'est une grande opportunité de chanter sur le plateau de l'Opéra d'Avignon. Merci à Sylvain, Cati, Aline, le groupe AKsAK, Zoé, Isabelle et la maîtresse. (Tessa)

J'ai beaucoup aimé chanter et je trouve que le groupe AKSAK nous accompagnait super bien. Le spectacle de samedi est passé trop vite. Ça va me manquer. (Léa)

J'ai beaucoup aimé. C'est passé trop vite. Sylvain va me manquer. Triste. (Gabriel C.)

Mes impressions sur la journée du 8 juin sont super. Car au début de la journée j'avais peur, mais en chantant je me sentais soulagée. Nous avons chanté pour des maternelles. Et j'adorais quand Zoé disait : "Car l'eau s'écoule et s'éloigne ». Et l'après-midi on a chanté pour des primaires. Et après le repas, un ciné dans une salle de théâtre Ouah ! (Inma)

J'ai trouvé ça trop bien. Quand ils ont ouvert le rideau rouge, j'avais les larmes aux yeux. J'ai adoré. (Mila)

C'était très bien. (Maé-Lou)

J'ai trouvé que c'était super bien. Je me suis beaucoup amusée. J'ai adoré chanter, c'était très bien. (Léna)

J'ai adoré chanter devant tout le monde, surtout devant ma famille. J'étais fier. Notre travail a porté ses fruits, nous avons fait une belle prestation. J'ai adoré ce spectacle et ma famille aussi. (Tom R.)

J'ai adoré tout ce qu'on a fait. Au début, j'étais angoissée, mais j'étais heureuse. Quand les rideaux se sont ouverts, mon angoisse est partie très loin ailleurs et j'ai chanté de tout mon cœur. (Emmy) 